\title{
TITLE:
}

\section{On Certain Vector Valued Siegel Modular Forms of Degree Two}

\author{
$\operatorname{AUTHOR}(\mathrm{S})$ :
}

Satoh, Takakazu

\section{CITATION:}

Satoh, Takakazu. On Certain Vector Valued Siegel Modular Forms of Degree Two. 数理解 析研究所講究録 1986, 583: 73-86

ISSUE DATE:

1986-02

URL:

http://hdl.handle.net/2433/99340

RIGHT: 


\title{
On Certain Vector Valued Siegel Modular \\ Forms of Degree Two
}

\author{
東工大 理 佐藤 孝和 (Takakazu Satoh)
}

\section{Introduction}

We explicitly construct vector valued siegel modular forms of degree two and the automorphic factor $\operatorname{det}^{k} \otimes S y m^{2}$ St for an even $k$ where st denotes the standard representation of $\mathrm{GL}(2, \mathrm{C})$. As an application, we prove some congruences between eigenvalues of Hecke operators. Details of this paper are contained in [12].

\section{Generalities}

Let $D=G / K$ be a tube domain where $G$ is a semi-simple Lie group and $K$ is its maximal compact subgroup. Let $C^{\infty}(G, V)$ be the set of V-valued $C^{\infty}$-functions on $G$. For a holomorphic representation $\rho$ of the complexification $K_{C}$ of $K$ and its representation space $V(p)$, we put

$$
C^{\infty}(G, V(\rho))_{\rho}=\left\{\begin{array}{l|l}
f \in C^{\infty}(G, V(\rho)) & \begin{array}{l}
f(g k)=\rho(k)^{-1} f(g) \\
\text { for all } g \in G \text { and } k \in K
\end{array}
\end{array}\right\} .
$$

Let 


$$
{ }^{g_{C}}=\mathbf{p}^{+} \oplus \mathbf{k}_{\mathbf{C}} \oplus \mathbf{p}^{-}
$$

be the Cartan decomposition where $\mathbf{g}$ and $\mathbf{k}$ denote the Lie algebras of $G$ and $K$ respectively and subscript $C$ stands for complexification. $A C^{\infty}$-function $f$ on $G$ is said to be of holo morphic type if it is annihilated by $\mathbf{p}^{-}$. Let $W$ be a finite dimensional $A d(K)$ invariant subspace of the symmetric algebra of $\mathbf{p}^{+}$and $\tau$ representation of $K$ on $W$ (by $\operatorname{Ad}(K)$ ). For $f \in C^{\infty}(G, V(\rho))_{\rho}$ and $X \in W$, we put

$$
D_{\tau} E(X)=r(X) E
$$

where $r(X)$ is right differential extended to the universal enveloping algebra of $\mathbf{g}_{\mathrm{C}}$. Then we have canonically

$$
D_{\tau} f \in C^{\infty}\left(G, V(\rho) \otimes W^{*}\right) \tau_{\rho}^{*}
$$

where $K$ acts on $W^{*}$ by contragradient representation $\tau^{*}$ of $\tau$. For a subgroup $\Gamma$ of $G$, the function $D_{\tau} f$ is left $\Gamma$-invariant if $f$ is left $\Gamma$-invariant. In general, $D_{\tau} f$ is not of holomorphic type. However we may cancel non-holomorphic term by taking suitable linear combination. All these things can be translated to the language of automorphic form on D. Using them in the case of $G=\operatorname{Sp}(2, R)$ and $W=\mathbf{p}^{+}$we construct holomorphic siegel modular forms of degree two and type $\operatorname{det}^{k} \otimes \mathrm{Sym}^{2} \mathrm{st}$. 
1. Construction of vector valued modular forms of type $(k, 2)$.

Let $\Gamma_{2}$ be the full Siegel modular group of degree 2 and $\mathrm{H}_{2}$ the Siegel upper half plane of degree 2 . Let $V(k, r)$ be a representation space of the holomorphic representation $\operatorname{det}^{k} \otimes S^{\prime} m^{r}$ st of $G L(2, C)$. A $C^{\infty}$-Siegel modular form $f$ of type $(k, r)$ and degree two is a $V(k, r)$ valued $C^{\infty}$-function on $H_{2}$ satisfying the equation

$$
E\left((A Z+B)(C Z+D)^{-1}\right)=\left(\operatorname{det}^{k} \otimes S y m^{r} S t\right)(C Z+D) E(Z)
$$

for all $M=\left[\begin{array}{ll}A & B \\ C & D\end{array}\right] \in \Gamma_{2}$ and for all $Z \in H_{2}$ and the usual growth rate condition (see Borel [2, $(7]$ ), which is satisfied for $f$ treated in this paper. We denote by $M_{k, r}^{\infty}\left(\Gamma_{2}\right)$ the c-vector space of all such functions. If $r=0$, the subscript $k, r$ is abbreviated as $k$ and type $(k, r)$ is mentioned as weight $k$ for simplicity. We also denote by $M_{k, r}\left(\Gamma_{2}\right)$ and $S_{k, r}\left(\Gamma_{2}\right)$ subspaces of $M_{k, r}^{\infty}\left(\Gamma_{2}\right)$ consisting of all holomorphic modular forms and all holomorphic cusp forms, respectively. Let $\mathrm{S}_{2}$ be the c-vector space of complex symmetric matrices of size two. The action of $\operatorname{GEGL}(2, \mathrm{C})$ defined by

$$
A \longrightarrow \operatorname{det}(G)^{k} G A^{t} G \quad\left(A \in S_{2}\right)
$$

is equivalent to $\operatorname{det}^{k} \otimes S y m^{2} S t$ where ${ }^{t} G$ is the transpose of $G$. Henceforth, we set $V(k, 2)=S_{2}$. For the variable $Z=\left[\begin{array}{ll}z_{1} & z_{3} \\ z_{3} & z_{2}\end{array}\right]$ on $H_{2}$ and $f \in M_{k}\left(\Gamma_{2}\right)=M_{k, 0}\left(\Gamma_{2}\right)$, we define the differential operator $\nabla=\nabla_{k}$ by 


$$
\nabla f=\frac{k}{2 \pi i}(2 i Y)^{-1} f+\frac{1}{2 \pi i} \frac{d}{d Z} f
$$

where

$$
\frac{d}{d z}=\left[\begin{array}{cc}
\partial_{1} & (1 / 2) \partial_{3} \\
(1 / 2) \partial_{3} & \partial_{2}
\end{array}\right] \text { with } \partial_{j}=\frac{\partial}{\partial z_{j}}
$$

and $Y=\frac{1}{2 i}(Z-\bar{Z})$. By Shimura $[15,(4.5)]$, we see that $\nabla f \in M_{k, 2}^{\infty}\left(\Gamma_{2}\right)$. For $f \in M_{k}\left(\Gamma_{2}\right)$ and $g \in M_{j}\left(\Gamma_{2}\right)$, we put

$$
[f, g]=\frac{1}{2 \pi i}\left[\frac{1}{j} f \frac{d}{d z} g-\frac{1}{k} g \frac{d}{d z} f\right] \text {. }
$$

By (1.1), we have

$$
[f, g]=\frac{1}{j} f \nabla g-\frac{1}{k} g \nabla f,
$$

so $[f, g] \in M_{k+j, 2}\left(\Gamma_{2}\right)$.

The dimension formula of $S_{k, r}\left(\Gamma_{2}\right)$ for $r=0$ and $k \geqq 4$ or $r \geqq 1$ and $k \geq 5$ is obtained by Tsushima [16, 17]. We use a method of Maass to evaluate dim $S_{k, 2}\left(\Gamma_{2}\right)$ for a small k.

Proposition 1 . Let $k \leqq 6$ be an integer. Then $\operatorname{dim} S_{k, 2}\left(\Gamma_{2}\right)=0$.

This proposition is proved by a method similar to Maass [9, pp. 189-196].

Recall that the graded $\mathrm{C}$-algebra $\underset{\mathrm{k}}{\oplus \mathrm{M}_{k}}\left(\Gamma_{2}\right)$ where $\mathrm{k}$ runs over even integers is generated over $c$ by four algebraically independent elements. (We understand that $M_{k}\left(\Gamma_{2}\right)=\{0\}$ for a negative $k$.) They are $\varphi_{4} \in M_{4}\left(\Gamma_{2}\right), \varphi_{6} \in M_{6}\left(\Gamma_{2}\right), \chi_{10} \in S_{10}\left(\Gamma_{2}\right)$ and $\chi_{12} \in S_{12}\left(\Gamma_{2}\right)$. For an odd $k$, we have $M_{k}\left(\Gamma_{2}\right)=\chi_{35} M_{k-35}\left(\Gamma_{2}\right)$ where $\chi_{35}$ is a cusp form of weight 35 . (See Igusa [5] and 
Maass [10].)

Theorem 2. For each even integer $k$, we have (as a c-vector space )

$$
\begin{aligned}
M_{k, 2}\left(\Gamma_{2}\right) & =M_{k-10}\left(\Gamma_{2}\right)\left[\varphi_{4}, \varphi_{6}\right] \oplus M_{k-14}\left(\Gamma_{2}\right)\left[\varphi_{4}, \chi_{10}\right] \\
& \oplus M_{k-16}\left(\Gamma_{2}\right)\left[\varphi_{4}, \chi_{12}\right] \oplus V_{k-16}\left(\Gamma_{2}\right)\left[\varphi_{6}, \chi_{10}\right] \\
& \oplus V_{k-18}\left(\Gamma_{2}\right)\left[\varphi_{6}, \chi_{12}\right] \oplus W_{k-22}\left(\Gamma_{2}\right)\left[\chi_{10}, \chi_{12}\right]
\end{aligned}
$$

and

$$
\begin{aligned}
S_{k, 2}\left(\Gamma_{2}\right) & =S_{k-10}\left(\Gamma_{2}\right)\left[\varphi_{4}, \varphi_{6}\right] \oplus M_{k-14}\left(\Gamma_{2}\right)\left[\varphi_{4}, \chi_{10}\right] \\
& \oplus M_{k-16}\left(\Gamma_{2}\right)\left[\varphi_{4}, \chi_{12}\right] \oplus V_{k-16}\left(\Gamma_{2}\right)\left[\varphi_{6}, \chi_{10}\right] \\
& \oplus V_{k-18}\left(\Gamma_{2}\right)\left[\varphi_{6}, \chi_{12}\right] \oplus W_{k-22}\left(\Gamma_{2}\right)\left[\chi_{10}, \chi_{12}\right]
\end{aligned}
$$

where

$$
\begin{aligned}
& v_{k}\left(\Gamma_{2}\right)=M_{k}\left(\Gamma_{2}\right) \cap \mathrm{c}\left[\varphi_{6}, \chi_{10}, \chi_{12}\right] \text { and } \\
& w_{k}\left(\Gamma_{2}\right)=M_{k}\left(\Gamma_{2}\right) \cap c\left[\chi_{10}, \chi_{12}\right] .
\end{aligned}
$$

Proof. (Outline.) The inclusion $\supset$ is clear in both (1.2) and (1.3). We show that subspaces appearing in the right hand side of (1.2) are mutually linearly independent. This is shown by the following lemma.

Lemma 3. Let $k$ be an integer. For $j=4,6,10$ and 12 , let $E_{j} \in M_{k-j}\left(\Gamma_{2}\right)$. If 


$$
f_{4} \frac{d}{d z} \varphi_{4}+f_{6} \frac{d}{d z} \varphi_{6}+f_{10} \frac{d}{d z} \chi_{10}+f_{12} \frac{d}{d z} \chi_{12}=0 \text {. }
$$

then we have

$$
f_{4}=f_{6}=f_{10}=f_{12}=0 \text {. }
$$

Using linear independency we show that the equality holds in (1.2). Let $d_{k}$ be the dimension of the the right hand side of (1.2). Then,

$$
\sum_{k=0}^{\infty} d_{k} T^{k}=\frac{T^{10}+T^{14}+2 T^{16}+T^{18}-T^{20}-T^{26}-T^{28}+T^{32}}{\left(1-T^{4}\right)\left(1-T^{6}\right)\left(1-T^{10}\right)\left(1-T^{12}\right)}
$$

where $T$ is an indeterminate. On the other hand, by Arakawa [1, Proposition 1.3] we have

$$
M_{k, 2}\left(\Gamma_{2}\right)=E_{k, 2}\left(\Gamma_{2}\right) \oplus S_{k, 2}\left(\Gamma_{2}\right)
$$

where $E_{k, 2}$ is the space of Eisenstein series of type $(k, 2)$ and

$$
\sum_{k=0}^{\infty} \operatorname{dim} E_{k, 2}\left(\Gamma_{2}\right) T^{k}=\frac{T^{10}}{\left(1-T^{4}\right)\left(1-T^{6}\right)} .
$$

By Tsushima [16, Theorem 4] (cf. Tsushima [17, Table 1]) and Proposition 1 we obtain

$$
\sum_{k: \text { even }} \operatorname{dim} S_{k, 2}\left(\Gamma_{2}\right) T^{k}=\frac{T^{14}+2 T^{16}+T^{18}+T^{22}-T^{26}-T^{28}}{\left(1-T^{4}\right)\left(1-T^{6}\right)\left(1-T^{10}\right)\left(1-T^{12}\right)} \text {. }
$$

Comparing (1.4), (1.5) and (1.6) we see that $d_{k}=\operatorname{dim} M_{k, 2}\left(\Gamma_{2}\right)$ for each even $k$, so the right hand side of (1.2) spans the left hand side. Noting $\operatorname{dim} E_{k, 2}\left(\Gamma_{2}\right)=\operatorname{dim} M_{k-10}\left(\Gamma_{2}\right)-\operatorname{dim} S_{k-10}\left(\Gamma_{2}\right)$, 
we have (1.3) by the same arguments.

Q.E.D.

A modular form $f \in M_{k, r}^{\infty}\left(\Gamma_{2}\right)$ is said to be an eigenform if $f$ is a non zero common eigen function of all Hecke operators. Let $f$ be an eigenform. We denote the eigenvalue of the $m$-th Hecke operator $T(m)$ by $\lambda(m, f)$ and put $Q(f)=Q(\lambda(m, f) \mid m \geqq 1)$. For a holomorphic function $f$ on $H_{n}$ satisfying $f(Z+S)=f(Z)$ for all $\mathrm{Z} \in \mathrm{H}_{\mathrm{n}}$ and all symmetric integral matrices $\mathrm{S}$ of size $\mathrm{n}$, we denote the Fourier expansion of $f$ by

$$
f(Z)=\sum_{N} a(N, f) \exp (2 \pi i \operatorname{Tr}(N Z))
$$

where $N$ runs over all semi-integral matrices and a( $N, f)$ stands for the Fourier coefficient of $f$ at $N$. For a subring $R$ of $C$, we put

$$
M_{k, 2}\left(\Gamma_{2}\right)_{R}=\left\{f \in M_{k, 2}\left(\Gamma_{2}\right) \mid a(N, f) \in M(2, R) \text { for all } N \geq 0\right\}
$$

and

$$
S_{k, 2}\left(\Gamma_{2}\right)_{R}=S_{k, 2}\left(\Gamma_{2}\right) \cap M_{k, 2}\left(\Gamma_{2}\right)_{R} \cdot
$$

Theorem 2 yields the following corollary.

Corollary 4. Let $f \in M_{k, 2}\left(\Gamma_{2}\right)$ be an eigenform for an even integer k. Then, $Q(f) / Q$ is a totally real finite extension, and the eigenvalues $\lambda(m, f)$ are algebraic integers for all $m \geq 1$. For a subring $R$ of $C$, the $R$ module $M_{k, 2}\left(\Gamma_{2}\right)_{R}$ is stable under $T(m)$ for all $\mathrm{m} \geqq 1$. 
Remark 5 . Let $R$ be a subring of $C$. For each odd integer $k \geq 39$, we see that $M_{k, 2}\left(\Gamma_{2}\right)_{R}$ is a non-zero $R$-submodule of $M_{k, 2}\left(\Gamma_{2}\right)$ and that $M_{k, 2}\left(\Gamma_{2}\right)_{R}$ is stable under $T(m)$ for all $m \geq 1$.

To prove congruences treated later, we construct a map from $M_{k, 2}\left(\Gamma_{2}\right)$ to $M_{k+2}^{\infty}\left(\Gamma_{2}\right)$ which commutes Hecke operators up to constants. Following Mass [8], we define a differential operator $\delta_{k}$ acting on a $C^{\infty}$-function $\mathrm{E}$ on $\mathrm{H}_{2}$ by

$$
\delta_{k} f=(2 \pi i)^{-2}|Y|^{-k+(1 / 2)}\left|\frac{d}{d Z}\right|\left(|Y|^{k-(1 / 2)} f\right) .
$$

By Harris $[3,1.5 .3], \delta_{k} \operatorname{maps} M_{k}^{\infty}\left(\Gamma_{2}\right)$ to $M_{k+2}^{\infty}\left(\Gamma_{2}\right)$. We define a subspace $\mathrm{PM}_{k}^{1}\left(\Gamma_{2}\right)$ of $M_{k}^{\infty}\left(\Gamma_{2}\right)$ by

$$
\begin{aligned}
\operatorname{PM}_{k}^{1}\left(\Gamma_{2}\right)= & M_{k}\left(\Gamma_{2}\right)+\delta_{k-2} M_{k-2}\left(\Gamma_{2}\right) \\
& +\left\{f \delta_{j} g \mid f \in M_{k-2-j}\left(\Gamma_{2}\right), g \in M_{J}\left(\Gamma_{2}\right)\right\}_{C}
\end{aligned}
$$

where \{ $\}$ stands for a C-linear span. The next theorem is essentially the particular case considered abstractly in Harris and Jakobsen [4, $\$ 1]$. But our result is so explicit that each Fourier coefficient can be computed effectively (and we can prove congruences).

Theorem 6. Let $F \in M_{k, 2}\left(\Gamma_{2}\right)$ for an even integer $k$. Then there exists the unique element $D(F)$ of $P M_{k+2}^{1}\left(\Gamma_{2}\right)$ satisfying the following conditions (a) and (b): 
(a) With respect to the Petersson inner product, $D(F)$ lies in the orthogonal complement of $\mathrm{S}_{k+2}\left(\Gamma_{2}\right)$ in $\mathrm{PM}_{k+2}^{1}\left(\Gamma_{2}\right)$.

(b) The function $H(F)$ defined by

$$
H(F)=D(F)-\frac{1}{2}|2 \pi Y|^{-1} \operatorname{Tr}(2 \pi Y F)
$$

is a holomorphic function having Fourier expansion of the following form

$$
H(F)(Z)=\sum_{N>0} a(N, H(F)) \exp (2 \pi i \operatorname{Tr}(N Z))
$$

where $\mathrm{N}$ runs over all positive definite semi-integral matrices of size two.

Moreover, if $F \in M_{k, 2}\left(\Gamma_{2}\right)$ is an eigenform, then $D(F) \in P M_{k+2}^{1}\left(\Gamma_{2}\right)$ is an eigenform satisfying

$$
\lambda(m, D(F))=m \lambda(m, F)
$$

for all $m \geqq 1$.

\section{Congruence formulas}

We prove some congruence formulas between eigenvalues of Hecke operators. Unfortunately, the method is not so systematic as that of serre [13]. In principle, this is done by comparison of Fourier coefficients. However on congruences between eigen functions of different type, say type $(k, 2)$ and weight $k+2$, we cannot compare them immediately. For this purpose, we use Theorem 6. Let $S_{k}\left(\Gamma_{1}\right)$ be the space of cusp forms of degree one and weight $k$. For a cusp form $f \in S_{k+2}\left(\Gamma_{1}\right)$, we denote by 
$[f]_{2} \in M_{k, 2}\left(\Gamma_{2}\right)$ the Klingen type Eisenstein series attached to $f$ defined by $[f]_{2}(Z)=E_{k, Z}(Z, f, Q)$ in the notation of Arakawa $[1,(1.4)]$. We denote by $\Delta_{16}$ the eigen cusp form of weight 16 normalized as $a\left(1, \Delta_{16}\right)=1$. For simplicity, we put $\eta_{14}=\left[\chi_{10}, \varphi_{4}\right]$. Using Theorem 2 we see that an eigen basis of $M_{14,2}\left(\Gamma_{2}\right)$ is $\left\{\left[\Delta_{16}\right]_{2}, \eta_{14}\right\}$, while an eigen basis of $S_{16}\left(\Gamma_{2}\right)$ is $\left\{\chi_{16}^{(+)}, \chi_{16}^{(-)}\right\}$where

$$
\chi_{16}^{( \pm)}=185 \cdot 4 \chi_{10} \varphi_{6}+(-128 \pm \sqrt{51349}) 12 \chi_{12} \varphi_{4} .
$$

respectively by Kurokawa $[6, \S 3]$.

Theorem 7. The following congruences hold for all $\mathrm{m} \geqq 1$ :

$$
\lambda\left(m, \eta_{14}\right) \equiv \lambda\left(m,\left[\Delta_{16}\right]_{2}\right) \bmod 373,
$$

and

$$
\mathrm{N}_{\mathrm{K} / \mathrm{Q}}\left[\mathrm{m} \lambda\left(\mathrm{m}, \eta_{14}\right)-\lambda\left[\mathrm{m}, \chi_{16}^{( \pm)}\right]\right] \equiv 0 \bmod 13
$$

where $K=Q(\sqrt{51349})$ and $N_{K / Q}$ is the norm map.

Proof. (Outline.) The proof of (2.1) is standard. By a numerical computation, we have

$$
\frac{1}{144}\left[\varphi_{6}, \varphi_{4}^{2}\right]=\left[\Delta_{16}\right]_{2}-\frac{403200}{373} \eta_{14} .
$$

Denominator 373 gives rise to the congruence (2.1). (Cf. Kurokawa[7, Theorem 1].) As to (2.2), we first compute $D\left(\eta_{14}\right)$. This shows

$$
N_{K / Q}\left(a\left(E, 138320 H\left(\eta_{14}\right)\right)-a\left[E, \chi_{16}^{( \pm)}\right]\right] \equiv 0 \bmod 13
$$


Using the uniqueness of Fourier coefficients we have

$$
N_{K / Q}\left[\left(\lambda\left(m, D\left(\eta_{14}\right)\right)-\lambda\left(m, \chi_{16}^{( \pm)}\right)\right) a\left(E, 138320 H\left(\eta_{14}\right)\right)\right] \equiv 0 \bmod 13
$$

which is equivalent to $(2.2)$ by Theorem 6 and $a\left(E, H\left(\eta_{14}\right)\right)=\frac{1}{130}$.

$$
\text { Q.E.D. }
$$

With respect to congruences of eigenvalues between eigen cusp forms of type $(k, 2)$ and weight $k$, we have the following general result. We denote by $\mathrm{Z}(\mathrm{E})$ the integer ring of $Q(E)$.

Theorem 8. Let $F \in S_{k}\left(\Gamma_{2}\right)$ be an eigenform. Let $\ell_{0}$ be a prime number dividing $k$ satisfying

$$
\begin{array}{ll}
\ell_{0} \neq 2,3,5 & \text { if } k \text { is even, } \\
\ell_{0} \neq 5,7 & \text { if } k \text { is odd. }
\end{array}
$$

Let $\ell$ be a prime ideal of $Z(F)$ lying above $\ell_{0}$. Then, there exists an eigenform $G \in S_{k, 2}\left(\Gamma_{2}\right)$ such that

$$
N_{K(G) / K}(\lambda(m, G)-m \lambda(m, F)) \equiv 0 \bmod \ell \text { for all } m \geqq 1
$$

where $K=Q(F)$ and $K(G)=K(\lambda(m, G) \mid m \geqq 1)$.

As an example (giving skeleton of the proof), let $\mathrm{F}=\chi_{14} \in \mathrm{S}_{14}\left(\Gamma_{2}\right), \mathrm{K}=0, \ell=7$ and $\mathrm{R}=\mathrm{Z}_{(7)}$. Here $\chi_{14}=\varphi_{4} \chi_{10}$ is the eigen cusp form of weight 14 . Then $G=\eta_{14}$ since $\operatorname{dim} S_{14,2}\left(\Gamma_{2}\right)=1$ and we have

$$
\lambda\left(m, \eta_{14}\right) \equiv m \lambda\left(m, \chi_{14}\right) \quad \bmod 7
$$

In this case we have moreover 


$$
\lambda\left(m, \eta_{14}\right) \equiv m \lambda\left(m, \chi_{14}\right) \quad \bmod 35
$$

using

$$
\nabla 4 \chi_{14}-\frac{7}{2} 4 \chi_{10} \nabla \varphi_{4}=-10 \cdot 4 \eta_{14}
$$

and $a\left(N, \varphi_{4}\right) \equiv 0 \bmod 240$ for all non-zero semi-integral $\mathrm{N}$.

Congruence (2.1) would be related to a special value of the second L-function of $\Delta_{1 \varepsilon}$. Let $f \in S_{k}\left(\Gamma_{1}\right)$ be an eigen form, $L_{2}(S, E)$ the second L-function attached to $E$ and $\langle E, E\rangle$ its Petersson inner product normalized as in Shimura [14, (2.1)]. Put

$$
L_{2}^{*}(s, f)=L_{2}(s, f)(2 \pi)^{-(2 s-k+2)} \Gamma(s) /\langle f, f\rangle .
$$

Then, $L_{2}^{*}(s, f)$ belongs to $Q(f)$ for an even integer $s$ with $k \leqq s \leqq 2 k-2$ by Zagier [18, Theorem 2]. Using this theorem we have

$$
L_{2}^{*}\left(28, \Delta_{16}\right)=\frac{2^{9} \cdot 373}{3^{2} 5^{2} 7^{2} 11}
$$

Here we note $28=2(k+r)-2-r$ with $k=14$ and $r=2$. More generally we expect that $L_{2}^{*}(2(k+r)-2-r, f)$ appears in the denominator of Fourier coefficients of $E_{k, r}\left(z, f, v_{0}\right)$ with suitable choice of $v_{0}$ in Arakawa [1, (1.4)]. We notice that the case $r=0$ is proved in Mizumoto [11]. (Cf. Kurokawa [7].)

The author would like to thank Prof. R. Tsushima for communicating his paper [17] before publication, and Prof. N. Kurokawa for his encouragement. 


\section{References}

1. T. Arakawa: Vector valued Siegel's modular forms of degree two and the associated Andrianov L-functions, Manuscripta Math., 44, 155-185, (1983).

2. A. Borel: Introduction to automorphic forms, Proc. Sympos. Pure Math., 9, 199-210, AMS, 1966.

3. M. Harris: Special values of zeta functions attached to Siegel modular forms, Ann. Sci. École Norm. Sup., 14, 77120 , (1981).

4. M. Harris and H. P. Jakobsen: Singular holomorphic representations and singular modular forms, Math. Ann., $259,227-244,(1982)$.

5. J. Igusa: On Siegel modular forms of genus two. II, Amer. J. Math., 86, 392-412, (1964).

6. N. Kurokawa: Examples of eigenvalues of Hecke operators on Siegel cusp forms of degree two, Invent. Math., 49, 149$165,(1978)$.

7. N. Kurokawa: Congruences between siegel modular forms of degree two, Proc. Japan Acad., 55A, 417-422, (1979).

8. H. Maass: Die Differentialgleichungen in der Theorie der Siegelschen Modulfunktionen, Math. Ann., 126, 44-68, (1953).

9. H. Maass: Siegel's Modular forms and Dirichlet Series, Lecture Notes in Mathematics, 216, Berlin-Heidelberg-New York: Springer, 1971.

10. H. Maass: Lineare Relationen für die Fourierkoeffizienten einiger Modulformen zweiten Grades, Math. Ann., 232, 163$175,(1978)$. 
11. S. Mizumoto: Fourier coefficients of generalized Eisenstein series of degree two. I, Invent. Math., 65, 115-135, (1981).

12. T. Satoh: On certain vector valued siegel modular forms of degree two, (preprint, 1985).

13. J.-P. Serre: Congruences et formes modulaires. Séminaire Bourbaki, Exp. 416 (June 1972), Lecture Notes in Mathematics, 317, 319-339, Berlin-Heidelberg-New York: Springer, 1973.

14. G. Shimura: The special values of the zeta functions associated with cusp forms, Comm. Pure Appl. Math., 29, 783$804,(1976)$.

15. G. Shimura: On the derivatives of theta functions and modular forms, Duke Math. J., 44, 365-387, (1977).

16. R. Tsushima: An explicit dimension formula for the spaces of generalized automorphic form with respect to $\operatorname{Sp}(2, \mathrm{Z})$, Proc. Japan Acad., 59A, 139-142, (1983).

17. R. Tsushima: An explicit dimension formula for the spaces of generalized automorphic form with respect to $\operatorname{Sp}(2, \mathrm{Z})$. (preprint)

18. D. Zagier: Modular forms whose Fourier coefficients involve zeta-functions of quadratic fields, Lecture Notes in Math., 627, 105-169, Berlin-Heidelberg-New York: springer, 1977. 